\title{
White matter correlates of the disorganized speech dimension in schizophrenia
}

\author{
Petra V. Viher ${ }^{1} \cdot$ Katharina Stegmayer $^{1} \cdot$ Stéphanie Giezendanner $^{1}$. \\ Andrea Federspiel $^{1} \cdot$ Stephan Bohlhalter $^{2,3} \cdot$ Roland Wiest $^{4} \cdot$ Werner Strik $^{1}$. \\ Sebastian Walther ${ }^{1}$
}

Received: 19 June 2016 / Accepted: 13 December 2016

(C) Springer-Verlag Berlin Heidelberg 2016

\begin{abstract}
Disorganized speech is related to functional abnormalities in schizophrenia. To test the association between formal thought disorders (FTDs) and white matter microstructure, we applied a behavioral rating and diffusion tensor imaging in 61 patients with schizophrenia spectrum disorders. The Bern Psychopathology Scale was used to rate the dimension of language abnormalities ranging from negative FTDs, basically unaltered speech, to positive FTDs. Tract-based spatial statistics indicated increased fractional anisotropy in left hemispheric pathways of the language system in patients with negative FTDs. Thus, altered white matter properties in relevant fiber tracts may represent vulnerability to specific formal thought disorders.
\end{abstract}

Keywords Formal thought disorders - Language system · Bern Psychopathology Scale · Diffusion tensor imaging . Tract-based spatial statistics

Petra V. Viher

petra.viher@puk.unibe.ch

1 Translational Research Center, University Hospital of Psychiatry, Bolligenstrasse 111, 3000 Bern 60, Switzerland

2 Department of Clinical Research, Inselspital, Bern, Switzerland

3 Neurology and Neurorehabilitation Center, Luzerner Kantonsspital, Lucerne, Switzerland

4 Support Center of Advanced Neuroimaging (SCAN), University Institute of Diagnostic and Interventional Neuroradiology, Inselspital, Bern, Switzerland

\section{Introduction}

Disorganized speech or formal thought disorders (FTDs) are prominent in many psychiatric conditions, including depression, mania, personality disorders and even in healthy controls, but have been mostly linked to schizophrenia [1,2]. Since Bleuler first described "loosening of associations" as a significant symptom in schizophrenia [3], FTDs are one of the hallmarks, affecting speech and language comprehension [4]. The prevalence of FTDs in schizophrenia ranges 5-91\%, with 50\% in the largest study in schizophrenia [5, 6].

FTDs in schizophrenia are linked to structural brain abnormalities predominantly in the left superior temporal gyrus (STG), angular gyrus, inferior operculum and orbitofrontal cortex [7-10], and to functional abnormalities in the left superior and middle temporal gyrus [7, 11, 12].

Despite clear associations of FTDs with aberrant structure and function of the cortex, the relationship between white matter and FTDs is poorly understood. For example, WM abnormalities correlated with functional MRI activation in the language network in schizophrenia patients [13]. Likewise, WM abnormalities in language-related pathways were related to linguistic deficits in childhood-onset schizophrenia [14]. Furthermore, aberrant WM properties of single fiber tracts were associated with language deficits in schizophrenia [15-17].

All these studies suggest a link of FTDs in schizophrenia with disturbed WM in cerebral language pathways. However, no study investigated the association of WM with FTDs, possibly owing to the nature of current FTDs rating scales. Factor analytic studies support a dimensional approach to FTDs, ranging from normal speech to FTDs [5]. Furthermore, FTDs may comprise more than one dimension [18]. Most frequently, studies reported a disorganized factor with incoherence and tangentiality and a negative factor of FTDs with poverty of speech and speech content [5]. Newer 
scales differentiate positive and negative FTDs and acknowledge the subjective experience of thought disorders [1, 19]. Indeed, positive and negative FTDs are associated with specific neuropsychological profiles and outcomes $[5,20]$.

Here, we applied the Bern Psychopathology Scale (BPS), which organizes schizophrenia symptoms in three neurobiologically informed dimensions, i.e., language, limbic and motor [19]. The symptom severity is rated on a Likert scale ranging from severe inhibition (negative FTDs) to severe disinhibition (positive FTDs). Positive FTDs include loose association and increased speech production while negative FTDs include reduced speech production, concretism and inhibited thinking/blocking [19]. The distinction of inhibited and disinhibited language symptoms in the BPS is supported by cluster analysis and differential associations with positive and negative syndrome ratings [21, 22]. Thus, the distinction of FTDs in a negative and positive pole may reveal biological correlates, because different mechanisms could underlie the two ends of severe FTDs. In other words: it is unlikely that one pathophysiological pathway would result in two opposing thought disorders, i.e., negative versus positive. Therefore, we hypothesize that the BPS language dimension will demonstrate a linear relationship with WM microstructure in the cerebral language system.

\section{Materials and methods}

\section{Subjects}

Sixty-one patients with schizophrenia (37 men, 24 women) were recruited at the inpatient and outpatient departments of the University Hospital of Psychiatry Bern, Switzerland. Inclusion criteria were diagnoses of schizophrenia, schizoaffective disorder or schizophreniform disorder according to the structured clinical interview (SCID) and DSM-5 criteria. Exclusion criteria were any substancerelated addiction other than nicotine, past or current medical or neurological condition, histories of head trauma with loss of consciousness or electroconvulsive treatment and specific exclusion criteria for magnetic resonance imaging (MRI) scans (e.g., metallic implants, pregnancy and claustrophobia).

The severity of each core domain of the BPS was rated on a seven-point scale ranging from -3 (inhibition) 0 (not present) to 3 (disinhibition) [19]. This bipolar structure of symptom dimensions has proved valid in schizophrenia and extends the positive-negative dichotomy of schizophrenia symptoms [21, 23-25]. Further assessment of schizophrenia symptoms included the Positive and Negative Syndrome Scale (PANSS) [26]. All but four patients received antipsychotics, and dosages were computed as chlorpromazine equivalents (CPZ) [27]. Demographic and clinical characteristics are summarized in Table 1. The protocol was approved by the local ethics committee, "Kantonale Ethikkomission Bern" (KEK-BE 025/13) and adhered to the declaration of Helsinki. All participants provided written informed consent.

\section{MRI acquisition}

Imaging was performed on a 3T MRI scanner (Siemens Magnetom Trio; Siemens Medical Solutions, Erlangen, Germany) with a 12-channel headcoil. For DTI measurements,

Table 1 Demographic and clinical characteristics

\begin{tabular}{|c|c|c|c|c|c|}
\hline \multirow[t]{2}{*}{ Variables } & Group $1(n=7)$ & Group $2(n=42)$ & Group $3(n=12)$ & \multirow{2}{*}{$\begin{array}{l}\text { One-way ANOVA } \\
p\end{array}$} & \multirow{2}{*}{$\begin{array}{l}\text { All patients }(n=61) \\
M(\mathrm{SD})\end{array}$} \\
\hline & \multicolumn{3}{|l|}{$M(\mathrm{SD})$} & & \\
\hline Age (years) & $41.5(13.2)$ & $35.9(11.4)$ & $39.3(9.8)$ & 0.387 & $37.3(11.3)$ \\
\hline Education (years) & $12.1(2.9)$ & $13.2(2.9)$ & $14.5(5.5)$ & 0.366 & $13.3(3.6)$ \\
\hline Duration of illness (years) & $11.8(15.0)$ & $10.7(11.6)$ & $15.2(11.7)$ & 0.536 & $11.7(12.0)$ \\
\hline Number of episodes & $7.0(9.9)$ & $5.6(6.2)$ & $9.1(8.5)$ & 0.337 & $6.5(7.2)$ \\
\hline PANSS-Pos & $13.7(5.4)$ & $15.7(5.9)$ & $22.3(6.7)$ & 0.003 & $16.8(6.5)$ \\
\hline PANSS-Neg & $24.3(4.7)$ & $18.3(5.9)$ & $15.2(3.8)$ & 0.003 & $18.4(5.9)$ \\
\hline PANSS-Total & $75.1(17.7)$ & $67.4(18.1)$ & $72.8(19.5)$ & 0.452 & $69.3(18.3)$ \\
\hline $\mathrm{CPZ}$ (mg) & $382.9(375.8)$ & $426.4(338.7)$ & $289.0(192.6)$ & 0.427 & $394.4(319.8)$ \\
\hline BPS language dimension & $-2.3(0.5)$ & $0.1(0.9)$ & $2.3(0.5)$ & 0.000 & $0.3(1.5)$ \\
\hline BPS affect dimension & $-0.6(1.3)$ & $-0.7(1.2)$ & $-0.2(1.4)$ & 0.369 & $-0.6(1.2)$ \\
\hline BPS motor dimension & $-1.6(0.5)$ & $-0.7(1.2)$ & $0.8(1.2)$ & 0.000 & $-0.5(1.3)$ \\
\hline
\end{tabular}

Group 1: patients with a BPS language score of -3 and -2 . Group 2: patients with a BPS language score of $-1,0$ and +1 . Group 3: patients with a BPS language score of +2 and +3

PANSS Positive and Negative Syndrome Scale, PANSS-Pos subscale for positive symptoms, PANSS-Neg subscale for negative symptoms, PANSS-Total total score of PANSS, CPZ chlorpromazine equivalents, $M$ mean, $S D$ standard deviation 
we used a spin echo planar imaging (EPI) sequence (59 slices, FOV $=256 \times 256 \mathrm{~mm}^{2}$, sampled on a $128 \times 128$ matrix, slice thickness $=2 \mathrm{~mm}$, gap between slices $=0 \mathrm{~mm}$, resulting in 2- $\mathrm{mm}^{3}$ isotopic voxel resolution) and TR/ $\mathrm{TE}=8000 / 92 \mathrm{~ms}$ covering the whole brain $(40 \mathrm{mT} / \mathrm{m}$ gradient, $6 / 8$ partial Fourier, GRAPPA factor 2, bandwidth $1346 \mathrm{~Hz} / \mathrm{Px})$. The axial slices were positioned in the plane parallel to the AC-PC line and measured along 42 directions with a $b=1300 \mathrm{~s} / \mathrm{mm}^{2}$. The sequence included 4 images without diffusion weighting (e.g., $b=0 \mathrm{~s} / \mathrm{mm}^{2}$; the first and every subsequent 12 th image). We used a rotationally invariant and balanced diffusion-encoding scheme over the unit sphere to generate the DTI data. Acquisition time was 6 min.

\section{Data analysis}

\section{Image processing}

DTI analyses were performed using the Functional Magnetic Resonance Imaging of the Brain's Diffusion Toolbox (FMRIB) Software Library (FSL, http://www.fmrib. ox.ac.uk/fsl), including the tract-based spatial statistics (TBSS) software [28, 29]. The image of each subject was first corrected for head movement and eddy currents (using "eddy-tool" of FSL). FA images were created by fitting a tensor model to the raw data (using "FDT"), and then, a brain extraction tool was used (using "BET-tool" of FSL) [30]. All subjects' FA data were coregistered to a $1-\mathrm{mm}^{3}$ Montreal Neurological Institute (MNI) standard space. The alignment was performed applying FMRIB's nonlinear image registration tool [31]. A mean FA image was prepared and thinned in order to create a mean FA skeleton for group comparisons. To prevent the inclusion of nonskeletal voxels, we used a FA threshold of 0.2. Each subject's aligned FA data were then projected onto the skeleton. The resulting data were subjected to voxel-wise between subject statistics.

\section{Statistical analysis}

Statistical analysis for WM microstructure was carried out with TBSS, applying a nonparametric approach with permutation test theory in a general linear model (GLM) design matrix [29]. Within the GLM framework, we examined the association between the language dimension and FA, with age, CPZ, and the ratings of the BPS affectivity and motor dimensions as covariates of no interest. Correction for multiple comparisons was achieved with threshold-free cluster enhancement (TFCE) correction and 5000 permutations [32]. A TFCE-corrected $p$ value $<0.05$ was considered as statistically significant. Localization was performed in clusters with more than 100 voxels using the Johns Hopkins University (JHU)-ICBM-DTI-81 WM labels atlas and the JHU-WM tractography atlas in MNI space $[33,34]$.

\section{Results}

FA values were significantly inverse associated with the BPS global language score $(r=-0.380, p=0.003$; see Fig. 1). The distinction in a positive and negative pole of the BPS score was crucial, as the same analysis between FA values and the BPS global language score did not show a significant correlation when neglecting the positive and negative signs $(r=0.120, p=0.356)$.

We detected a negative linear association between the BPS global language score and WM microstructure in
Fig. 1 a Correlation of white matter microstructure and language dimension of the BPS. The TBSS image shows the negative correlation between the language dimension of the BPS and FA values within the areas indicated in red at $p<0.05$ corrected for multiple comparison. Covariates included age, CPZ, BPS ratings of affectivity and motor behavior. $Z$ indicates the coordinates of the image slices in $\mathrm{mm}$. b Correlation of mean FA of significant regions with the score of the language dimension of the BPS

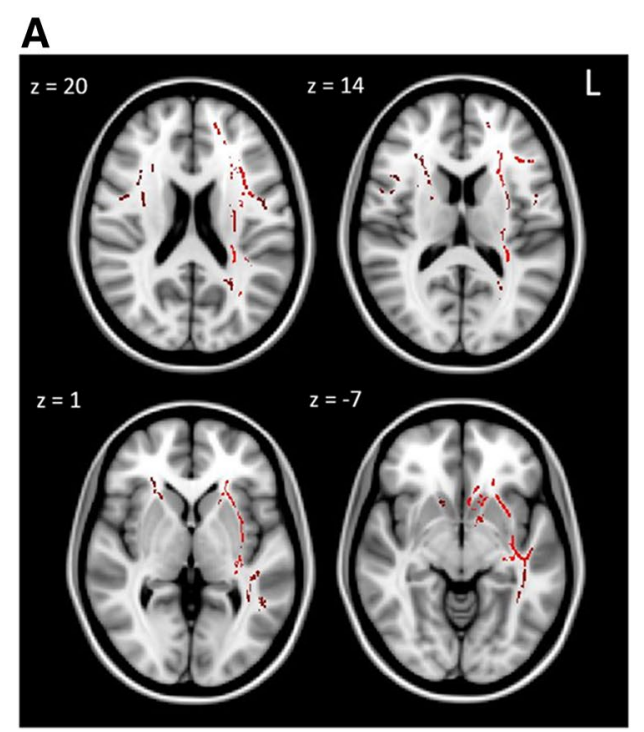

B

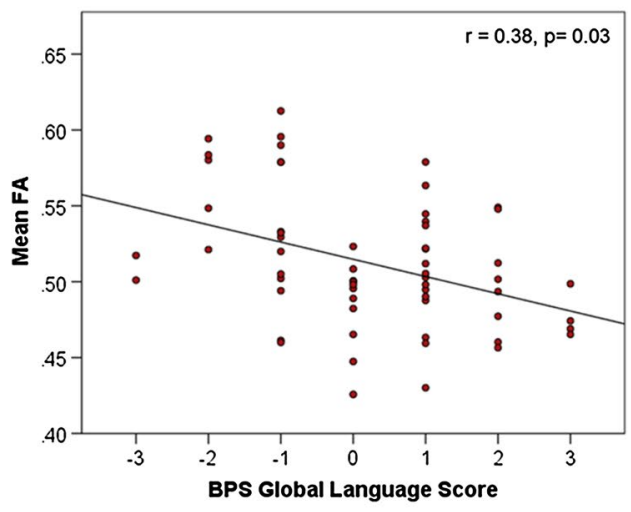


Table 2 Location of significant correlations between white matter microstructure (FA) and the language dimension of the BPS

\begin{tabular}{|c|c|c|c|c|c|}
\hline \multirow{2}{*}{$\begin{array}{l}\text { Location } \\
\text { FA (negative linear relationship) }\end{array}$} & \multicolumn{3}{|c|}{$\begin{array}{l}\text { Center of gravity ( } \mathrm{mm} \text { coordi- } \\
\text { nates) }\end{array}$} & \multirow[t]{2}{*}{ Cluster size } & \multirow[t]{2}{*}{$p$} \\
\hline & $\mathrm{X}$ & $\mathrm{Y}$ & $\mathrm{Z}$ & & \\
\hline Body of corpus callosum & -5.4 & -9.6 & 30.4 & 635 & 0.044 \\
\hline Splenium of corpus callosum & -8.5 & -42.6 & 24.2 & 307 & 0.047 \\
\hline Anterior limb of internal capsule $\mathrm{R}$ & 20.9 & 11.8 & 9.0 & 199 & 0.049 \\
\hline Retrolenticular part of internal capsule $\mathrm{L}$ & -30.8 & -30.1 & 7.5 & 365 & 0.042 \\
\hline Anterior corona radiata $\mathrm{R}$ & 25.5 & 25.0 & 8.0 & 303 & 0.048 \\
\hline Anterior corona radiata $\mathrm{L}$ & -23.4 & 23.6 & 9.6 & 450 & 0.042 \\
\hline Superior corona radiata $\mathrm{L}$ & -23.4 & -10.6 & 28.3 & 262 & 0.045 \\
\hline Posterior corona radiata $\mathrm{R}$ & 21.8 & -44.7 & 31.9 & 105 & 0.049 \\
\hline Posterior corona radiata $\mathrm{L}$ & -24.0 & -37.5 & 27.4 & 317 & 0.042 \\
\hline Posterior thalamic radiation $\mathrm{L}$ & -34.5 & -46.5 & 6.5 & 257 & 0.046 \\
\hline External capsule L & -29.6 & 2.7 & -0.4 & 799 & 0.043 \\
\hline Superior longitudinal fascicle $\mathrm{R}$ & 34.7 & -2.2 & 23.5 & 191 & 0.049 \\
\hline Superior longitudinal fascicle $\mathrm{L}$ & -36.8 & -25.5 & 28.2 & 678 & 0.042 \\
\hline Anterior thalamic radiation $\mathrm{R}$ & 21.1 & 15.7 & 8.1 & 120 & 0.048 \\
\hline Anterior thalamic radiation $\mathrm{L}$ & -22.3 & 23.2 & 9.5 & 110 & 0.042 \\
\hline Corticospinal tract $\mathrm{L}$ & -22.8 & -25.5 & 39.3 & 154 & 0.043 \\
\hline Forceps minor & -15.1 & 46.5 & 15.0 & 173 & 0.045 \\
\hline Inferior fronto-occipital fascicle $\mathrm{R}$ & 25.7 & 26.8 & 5.0 & 178 & 0.048 \\
\hline Inferior fronto-occipital fascicle $\mathrm{L}$ & -33.0 & -9.4 & -2.1 & 727 & 0.043 \\
\hline Inferior longitudinal fascicle $\mathrm{L}$ & -41.5 & -30.3 & -7.0 & 270 & 0.045 \\
\hline Uncinate fascicle $\mathrm{L}$ & -28.0 & 10.9 & -8.0 & 113 & 0.041 \\
\hline
\end{tabular}

predominantly left hemispheric clusters of the temporal and frontal lobe (see Fig. 1; Table 2). Higher FA values were associated with more BPS language inhibition in important tracts for language processing, such as the uncinate fascicle, the superior and inferior longitudinal fascicle and the inferior fronto-occipital fascicle $(p<0.05$, corrected for multiple comparisons).

\section{Discussion}

This is the first study to demonstrate a direct link between WM abnormalities in a predominantly left fronto-temporal language network and FTDs in schizophrenia. The fiber tracts associated with FTDs included the left uncinate fascicle, superior and inferior longitudinal fascicle and the inferior fronto-occipital fascicle.

Our findings within WM are in line with the existing literature, linking FTDs in schizophrenia to structural and functional brain abnormalities in the language system [7, $8,11,12]$. Previous studies demonstrated associations of WM properties with semantic processing [16], a disorganized thoughts factor [15] and a broad clinical factor including several positive symptoms [17]. For example, the left uncinate fascicle connects Broca's region with the temporal lobe [35]. In fact, language performance such as semantic tasks was shown to rely on uncinate WM integrity [35-37]. Likewise, FTDs severity in schizophrenia was associated with functional deviation of the language network in Broca's area [12]. Thus, WM abnormalities in the uncinate fascicle may contribute to poor integrity of the language network and consequently to FTDs.

However, there was no simple correlation with FTDs severity in the BPS when collapsing positive and negative FTDs. In contrast, the linear brain-behavior association with WM microstructure was only detected with the BPS language score ranging from inhibition (negative FTDs) to normal speech (no FTDs) and disinhibition (positive FTDs). Particularly, patients with behavioral inhibition of language had highest FA values, suggesting that structural specialization of language tracts with increased FA may increase vulnerability for negative FTDs. In contrast, lower FA in language tracts increases vulnerability to positive FTDs. Thus, the finding indicates distinct pathobiology of positive and negative FTDs, regarding the association of WM abnormalities and aberrant behavior.

Some limitations of this study require discussion. Age may impact frontal and parietal WM structure [38-40], 
but the effect of other variables, such as antipsychotic treatment, is still under debate [41-43]. Nevertheless, we included both variables as covariates of no interest into our analysis. In addition, we included the BPS affect and motor dimensions as covariates to rule out any unspecific effects of schizophrenia syndrome severity.

In conclusion, we found a direct link between a dimension of FTDs in schizophrenia and disturbed WM in language-related pathways. Further studies need to address the functional consequences of aberrant WM in language pathways at the level of cerebral network activity.

Acknowledgements This study received funding from the BangerterRhyner Foundation (to Sebastian Walther) and the Swiss National Science Foundation (SNF grant 152619/1 to Sebastian Walther, Andrea Federspiel and Stephan Bohlhalter).

\section{Compliance with ethical standards}

Conflict of interest The authors have declared that there are no conflicts of interest in relation to the subject of this study.

\section{References}

1. Kircher $\mathrm{T}$ et al (2014) A rating scale for the assessment of objective and subjective formal thought and language disorder (TALD). Schizophr Res 160(1-3):216-221

2. Andreasen NC, Grove WM (1986) Thought, language, and communication in schizophrenia: diagnosis and prognosis. Schizophr Bull 12(3):348-359

3. Bleuler E (1911) Dementia praecox oder Gruppe der Schizophrenien. Deuticke, Leipzig

4. Heckers S et al (2013) Structure of the psychotic disorders classification in DSM-5. Schizophr Res 150(1):11-14

5. Roche E et al (2015) The epidemiology and associated phenomenology of formal thought disorder: a systematic review. Schizophr Bull 41(4):951-962

6. Breier A, Berg PH (1999) The psychosis of schizophrenia: prevalence, response to atypical antipsychotics, and prediction of outcome. Biol Psychiatry 46(3):361-364

7. Horn $\mathrm{H}$ et al (2009) Structural and metabolic changes in language areas linked to formal thought disorder. Br J Psychiatry 194(2):130-138

8. Palaniyappan L et al (2015) Structural correlates of formal thought disorder in schizophrenia: an ultra-high field multivariate morphometry study. Schizophr Res 168(1-2):305-312

9. Sans-Sansa B et al (2013) Association of formal thought disorder in schizophrenia with structural brain abnormalities in languagerelated cortical regions. Schizophr Res 146(1-3):308-313

10. Horn $\mathrm{H}$ et al (2010) Gray matter volume differences specific to formal thought disorder in schizophrenia. Psychiatry Res 182(2):183-186

11. Kircher TT et al (2001) Neural correlates of formal thought disorder in schizophrenia: preliminary findings from a functional magnetic resonance imaging study. Arch Gen Psychiatry 58(8):769-774

12. Horn $\mathrm{H}$ et al (2012) Semantic network disconnection in formal thought disorder. Neuropsychobiology 66(1):14-23

13. Leroux E et al (2013) Functional and white matter abnormalities in the language network in patients with schizophrenia: a combined study with diffusion tensor imaging and functional magnetic resonance imaging. Schizophr Res 150(1):93-100

14. Clark K et al (2012) White matter integrity, language, and childhood onset schizophrenia. Schizophr Res 138(2-3):150-156

15. Asami $\mathrm{T}$ et al (2013) Abnormalities of middle longitudinal fascicle and disorganization in patients with schizophrenia. Schizophr Res 143(2-3):253-259

16. Kubicki M et al (2011) Stochastic tractography study of Inferior Frontal Gyrus anatomical connectivity in schizophrenia. Neuroimage 55(4): 1657-1664

17. Phillips OR et al (2009) Fiber tractography reveals disruption of temporal lobe white matter tracts in schizophrenia. Schizophr Res 107(1):30-38

18. Cuesta MJ, Peralta V (2011) Testing the hypothesis that formal thought disorders are severe mood disorders. Schizophr Bull 37(6):1136-1146

19. Strik W et al (2010) The Bern Psychopathology Scale for the assessment of system-specific psychotic symptoms. Neuropsychobiology 61(4):197-209

20. Nagels A et al (2016) Distinct neuropsychological correlates in positive and negative formal thought disorder syndromes: the thought and language disorder scale in endogenous psychoses. Neuropsychobiology 73(3):139-147

21. Lang FU et al (2015) Factor structure of the Bern Psychopathology Scale in a sample of patients with schizophrenia spectrum disorders. Eur Psychiatry 30(7):880-884

22. Lang FU et al (2016) Dimensional approaches to schizophrenia: a comparison of the Bern psychopathology scale and the fivefactor model of the Positive and Negative Syndrome Scale. Psychiatry Res 239:284-290

23. Lang FU et al (2015) Subtyping schizophrenia: a comparison of positive/negative and system-specific approaches. Compr Psychiatry $61: 115-121$

24. Bracht $\mathrm{T}$ et al (2012) Comparison of objectively measured motor behavior with ratings of the motor behavior domain of the Bern Psychopathology Scale (BPS) in schizophrenia. Psychiatry Res 198(2):224-229

25. Schoretsanitis G et al (2016) Keep at bay!-abnormal personal space regulation as marker of paranoia in schizophrenia. Eur Psychiatry 31:1-7

26. Kay SR, Fiszbein A, Opler LA (1987) The Positive and Negative Syndrome Scale (PANSS) for schizophrenia. Schizophr Bull 13(2):261-276

27. Woods SW (2003) Chlorpromazine equivalent doses for the newer atypical antipsychotics. J Clin Psychiatry 64(6):663-667

28. Smith SM et al (2004) Advances in functional and structural MR image analysis and implementation as FSL. Neuroimage 23(Suppl 1):S208-S219

29. Smith SM et al (2006) Tract-based spatial statistics: voxelwise analysis of multi-subject diffusion data. Neuroimage 31(4):1487-1505

30. Smith SM (2002) Fast robust automated brain extraction. Hum Brain Mapp 17(3):143-155

31. Andersson JLR, Jenkinson M, Smith S (2007) Non-linear optimisation FMRIB Technical Report TR07JA1

32. Smith SM, Nichols TE (2009) Threshold-free cluster enhancement: addressing problems of smoothing, threshold dependence and localisation in cluster inference. Neuroimage 44(1):83-98

33. Mazziotta $\mathbf{J}$ et al (2001) A probabilistic atlas and reference system for the human brain: International Consortium for Brain Mapping (ICBM). Philos Trans R Soc Lond B Biol Sci 356(1412):1293-1322

34. Mori S, Wakana S, Zijl PCM (2005) MRI atlas of human white matter. Elsevier, Amsterdam

35. Catani $\mathrm{M}$ et al (2013) A novel frontal pathway underlies verbal fluency in primary progressive aphasia. Brain 136(Pt 8):2619-2628 
36. Harvey DY et al (2013) Neuropsychological evidence for the functional role of the uncinate fasciculus in semantic control. Neuropsychologia 51(5):789-801

37. Paldino MJ, Hedges K, Zhang W (2014) Independent contribution of individual white matter pathways to language function in pediatric epilepsy patients. Neuroimage Clin 6:327-332

38. Davis SW et al (2009) Assessing the effects of age on long white matter tracts using diffusion tensor tractography. Neuroimage 46(2):530-541

39. Gunning-Dixon FM et al (2009) Aging of cerebral white matter: a review of MRI findings. Int J Geriatr Psychiatry 24(2):109-117

40. Konopaske GT et al (2008) Effect of chronic antipsychotic exposure on astrocyte and oligodendrocyte numbers in macaque monkeys. Biol Psychiatry 63(8):759-765
41. Kanaan R et al (2009) White matter microstructure in schizophrenia: effects of disorder, duration and medication. Br J Psychiatry 194(3):236-242

42. Peters BD, Blaas J, de Haan L (2010) Diffusion tensor imaging in the early phase of schizophrenia: what have we learned? J Psychiatr Res 44(15):993-1004

43. Monji A, Kato T, Kanba S (2009) Cytokines and schizophrenia: microglia hypothesis of schizophrenia. Psychiatry Clin Neurosci 63(3):257-265 